\title{
Performance Evaluation of the Aptima Assays in Comparison with the cobas 6800 Assays for the Detection of HIV-1, HBV, and HCV in Clinical Samples
}

\author{
Younhee Park (i), M.D., Ph.D. ${ }^{1}$, Juhye Roh $\mathbb{1 0}$, M.D., Ph.D. ${ }^{2}$, and Sinyoung Kim (), M.D., Ph.D. ${ }^{1}$ \\ ${ }^{1}$ Department of Laboratory Medicine, Severance Hospital, Yonsei University College of Medicine, Seoul, Korea; ${ }^{2}$ Department of Laboratory Medicine, Hallym \\ University Sacred Heart Hospital, Anyang, Korea
}

Background: Accurate and consistent viral load (VL) quantitation of HIV type 1 (HIV-1), hepatitis $B$ virus (HBV), and hepatitis $C$ virus (HCV) is important for diagnosis and clinical monitoring. Assay results have to be concordant and compatible across laboratories. We evaluated the performance of three Aptima assays (Hologic, San Diego, CA, USA) and compared their VL values with corresponding cobas 6800 assay (Roche Diagnostics, Mannheim, Germany) results, using 840 clinical samples.

Methods: The correlation between VL results obtained using the two assays was evaluated in terms of analytical sensitivity, precision/reproducibility, linearity, and cross-reactivity. Agreement rates were determined using kappa statistics. The overall agreement of VL values was examined using Passing-Bablok regression analysis.

Results: All CVs were within 5\%; the assays had good precision for detecting all three viruses. The linearity of quantitation assessed using three AccuSpan linearity panels (Seracare, Milford, MA, USA), was excellent for the Aptima assays. For HIV-1 and HCV, the results of both assays showed excellent agreement ( $\kappa=0.89$ and 0.90 , respectively) while for HBV, the results showed good agreement $(\kappa=0.69)$. For analytical sensitivity, the VLS required for a $100 \%$ detection rate of HIV-1, HBV, and HCV were 20 copies $/ \mathrm{mL}, 7.5 \mathrm{IU} / \mathrm{mL}$, and $5.0 \mathrm{IU} / \mathrm{mL}$, respectively. The results for HIV-1, HBV, and HCV obtained using both assays correlated strongly $\left(R^{2}=0.97,0.93\right.$, and 0.95 , respectively).

Conclusions: The cobas 6800 and Aptima assays, with fully automated and high-throughput molecular platforms for HIV-1, HBV, and HCV VL measurements, show good analytical performance and a strong correlation between results. The study results suggest that the assays can be used interchangeably for long-term monitoring of chronic infections.

Key Words: HIV, Hepatitis B virus, Hepatitis C virus, Viral load, Aptima assay, cobas 6800 assay, Performance, Agreement, Analytical sensitivity
Received: March 4, 2021

Revision received: June 6, 2021

Accepted: January 28, 2022

\section{Corresponding author:}

Sinyoung Kim, M.D., Ph.D. Department of Laboratory Medicine, Severance Hospital, Yonsei University College of Medicine, 50-1 Yonsei-ro, Seodaemun-gu, Seoul 03722, Korea Tel: +82-2-2228-2452 Fax: +82-2-2227-8353 E-mail: sykim@yuhs.ac

\section{INTRODUCTION}

Viral load (VL) quantitation of HIV type 1, hepatitis B virus (HBV), and hepatitis $\mathrm{C}$ virus (HCV) is a part of the standard protocol for diagnosis, clinical monitoring, and treatment management [1-8]. In patients infected with HIV-1, the VL should be measured at diagnosis and at the initiation of antiretroviral therapy, and regularly between 6 and 12 months thereafter. Treatment success can be judged based on a VL remaining $<50$ copies/mL [9-11]. For HBV infections, several guidelines recommend monitoring the VL using highly sensitive nucleic acid amplification technologies every three months until the HBV DNA is undetectable, and 
every three to six months thereafter to detect persistent viremia and virological breakthrough $[3,7]$. HCV RNA detection and VL quantification should be performed using a sensitive assay with a lower limit of detection (LOD) of $15 \mathrm{IU} / \mathrm{mL}$. The endpoint of therapy is undetectable HCV RNA for 12-24 weeks after the end of treatment [5].

The assays used for monitoring VLs of HIV-1, HBV, or HCV must have a high sensitivity and accuracy. Infections with these viruses are chronic and require long-term treatment and monitoring, during which VL measurements may be performed at different laboratories or using different reagents and analyzers within the same laboratory. Providing results that are consistent, concordant, and interchangeable across laboratories and assays is necessary [11-13]. Various assays for the quantitation of HIV-1, HBV, and HCV in plasma or serum are commercially available, but reportedly have variable performance [9, 14]. Most of these assays use quantitative real-time PCR technology. The recently developed Aptima assay (Hologic, San Diego, CA, USA) involves three steps: target capture, target amplification via transcription-mediated amplification (TMA), and real-time detection of the amplicons using fluorescent probes; all these processes are automated and performed in a single tube on the Panther platform (Hologic). Previous comparative performance studies have examined quantitative real-time PCR technology-based assays against the Aptima assay [9, 11, 14-30]. However, few studies have compared the assays available for the fully automated, high-throughput molecular platforms, Roche cobas 6800 (Roche Diagnostics, Mannheim, Germany) and Panther [9, 11, 31, 32]. We evaluated the interchangeability of assay results for long-term monitoring of chronic infections to compare the performance of the Aptima and cobas 6800 assays, using samples sufficient for the determination of HIV-1, HBV, and HCV VL values. This is the first study to analyze more than 100 positive samples representing a sufficient analytical range for HIV-1, HBV, and HCV detection commonly used in clinical laboratories.

\section{MATERIALS AND METHODS}

\section{Samples}

Blood samples were collected from patients with HIV-1, HBV, or $\mathrm{HCV}$ infection. Plasma was obtained from these samples treated with EDTA to prevent coagulation. In total, 301, 300, and 239 plasma samples assayed using cobas 6800 between June and August 2020 were retested using the HIV-1, HBV, and HCV Aptima assays, respectively. The samples were stored at $-70^{\circ} \mathrm{C}$ for up to four months before use in the Aptima assays. The re- search involving human samples complied with all the relevant national regulations, institutional policies, and the tenets of the Helsinki Declaration (as revised in 2013), and was approved by the Institutional Review Board of the Severance Hospital, Seoul, Korea (IRB No.: 1-2020-0019). The requirement for informed consent was waived due to the retrospective nature of this study, provided that all patient data were anonymized and that the study involved samples already available rather than prospectively collected.

\section{Assay characteristics and evaluation}

The characteristics of each assay are presented in Table 1. All the instruments and assays were used by trained operators in accordance with the manufacturers' instructions.

The imprecision was evaluated according to CLSI guideline EP15-A3, and the inter- and intra-assay precision was determined [33]. Low- and high-concentration samples of positive control materials provided by each manufacturer were used. The control materials were measured in two replicates twice daily for 10 days to assess inter-assay precision. For intra-assay precision assessment, 20 replicates of high- and low-concentration positive control materials were used.

The linearity was evaluated according to CLSI guideline EP06A, using the AccuSpan HIV-1 RNA Linearity Panel (Seracare, Milford, MA, USA; 2410-0221/Batch \#01383902) with a concentration range of 2.10-8.35 log copies/mL, the AccuSpan HBV DNA Linearity Panel (PHD802 [2410-0162]/Batch \#10439611) with a concentration range of 1.74-8.40 $\log \mathrm{IU} / \mathrm{mL}$, and the AccuSpan HCV RNA Linearity Panel (PHW805 [2410-0166]/Batch \#10338244) with a concentration range of 1.26-7.34 log IU/mL [34]. Each panel item was measured twice, and the mean values obtained were compared with the expected values indicated in the panel datasheets. Linear-fit and second- and third-order polynomial regression analyses were performed. A regression equation was obtained using the observed and expected VL values as dependent and independent variables, respectively, from the best-fitting model.

The clinical samples from the patients with HIV-1, HBV, or $\mathrm{HCV}$ infection requiring VL monitoring were assayed with the routinely used cobas 6800 assays in parallel with the Aptima assays of the Panther platform. The assay results were categorized as "Target not detected" (TND), "below the lower limit of quantitation" (<LLOQ), or "quantitated" based on the LLOQ value of each assay. Quantitative results from both assays were evaluated for correlation. HIV-1 drug resistance mutation and $\mathrm{HCV}$ genotyping data were collected for the relevant samples. 
Park Y, et al.

Aptima vs. cobas 6800 for HIV-1, HBV, and HCV

Table 1. Characteristics of the Panther and cobas 6800 automated molecular platforms

\begin{tabular}{|c|c|c|c|}
\hline & & Panther* & Cobas $6800^{\dagger}$ \\
\hline \multicolumn{2}{|l|}{ Principle } & Transcription-mediated amplification & Real-time quantitative PCR \\
\hline \multicolumn{2}{|l|}{ Assay type } & Fully automated, random-access system & Fully automated, batched system \\
\hline \multirow[t]{3}{*}{ Targets } & HIV-1 & Pol, LTR regions & Gag gene and LTR region \\
\hline & HBV & Pol, surface (S) gene & Pol, precore region \\
\hline & $\mathrm{HCV}$ & $5^{\prime}$ UTR & $5^{\prime}$ UTR \\
\hline \multirow[t]{3}{*}{ Sample type } & HIV-1 & Plasma, DBS & Plasma, PSC \\
\hline & HBV & Plasma, serum & Plasma, serum \\
\hline & HCV & Plasma, serum & Plasma, serum \\
\hline \multicolumn{2}{|l|}{ Sample volume } & $1200 \mu \mathrm{L}$ (primary tube), $700 \mu \mathrm{L}$ (secondary tube) & $650 \mu \mathrm{L}$ \\
\hline \multicolumn{2}{|c|}{ Sample processing volume } & $500 \mu \mathrm{L}$ & $500 \mu \mathrm{L}$ \\
\hline \multirow[t]{3}{*}{ Limit of detection } & HIV-1 & 12 copies/mL & 13.2 copies $/ \mathrm{mL}$ \\
\hline & HBV & $4.8 \mathrm{IU} / \mathrm{mL}$ (plasma), $5.9 \mathrm{IU} / \mathrm{mL}$ (serum) & $2.7 \mathrm{IU} / \mathrm{mL}$ (plasma), $2.4 \mathrm{IU} / \mathrm{mL}$ (serum) \\
\hline & $\mathrm{HCV}$ & $3.9 \mathrm{IU} / \mathrm{mL}$ (plasma), $3.4 \mathrm{IU} / \mathrm{mL}$ (serum) & $8.5 \mathrm{IU} / \mathrm{mL}$ (plasma), $9.6 \mathrm{IU} / \mathrm{mL}$ (serum) \\
\hline \multirow[t]{3}{*}{ Linear assay range } & HIV-1 & $30-1 \times 10^{6}$ copies $/ \mathrm{mL}$ & $20-1 \times 10^{7}$ copies $/ \mathrm{mL}$ \\
\hline & HBV & $10-1 \times 10^{9} \mathrm{IU} / \mathrm{mL}$ & $10-1 \times 10^{9} \mathrm{IU} / \mathrm{mL}$ \\
\hline & HCV & $10-1 \times 10^{8} \mathrm{IU} / \mathrm{mL}$ & $15-1 \times 10^{8} \mathrm{IU} / \mathrm{mL}$ \\
\hline \multicolumn{2}{|c|}{ Time to results from start } & $\sim 210$ minutes for five results & $\sim 180$ minutes for 96 results \\
\hline \multicolumn{2}{|l|}{ Result timeline } & five results every 5 minutes & 93-minute average time between plates \\
\hline
\end{tabular}

*The Panther platform was used for the Aptima assays; ${ }^{\dagger}$ The Cobas 6800 platform was used for the cobas 6800 assays.

Abbreviations: Pol, polymerase; LTR, long terminal repeat; HIV, Human immunodeficiency virus; HBV, hepatitis B virus; HCV, hepatitis C virus; 5’ UTR, 5’ untranslated region; DBS, dried blood spot; PSC, plasma separation card.

HIV-1 drug resistance mutation was assessed using a sequencebased typing method and interpreted according to the HIVdb Program of the HIV Drug Resistance Database provided by Stanford University [35]. For HCV genotyping, the Roche cobas HCV GT kit was used on the Roche cobas 4800 automated platform. HBV genotyping was not performed because HBV genotype C prevails predominantly among chronic carriers of the virus in Korea, irrespective of their clinical stages of liver disease and geographic origin.

To assess the analytical sensitivity of the Aptima assays, the clinical samples were diluted with virus-negative plasma to obtain target concentrations of 20,30 , and 40 copies/mL for HIV1 , and of $5,7.5,10$, and $15 \mathrm{IU} / \mathrm{mL}$ for HBV and HCV, based on the cobas 6800 results. Twenty replicates of each dilution were assayed within the same run. The lowest concentrations yielding a $100 \%$ detection rate and $100 \%$ quantitation rate were compared with the LOD and LLOQ reported by the manufacturer.

The potential cross-reactivity with other viruses was assessed using two independent samples with detected HIV-1, HBV, HCV, cytomegalovirus, Epstein-Barr virus, BK virus, and herpes simplex virus, but not the virus to be measured by the assay.

\section{Statistical analysis}

All statistical analyses were performed using the Analyse-it software, version 4.65 (Analyse-it Software, Leeds, UK). The agreement rates between the two assays were determined using the kappa statistics with $95 \%$ confidence intervals $(\mathrm{Cls}$ ) (with $\kappa$ values of $0.001-0.2,0.201-0.4,0.401-0.6,0.601-0.8$, and 0.801-0.999 representing slight, fair, moderate, substantial, and excellent agreement, respectively). The overall correlation of VL values for samples quantitated with both assays was examined pairwise using Passing-Bablok regression analysis, which included the calculation of a correlation coefficient $\left(R^{2}\right)$, as well as Bland-Altman analysis, which included the calculation of the mean difference and $95 \% \mathrm{Cl}$ of the assay agreement. $\mathrm{HCV}$ values stratified according to the genotype were examined using ANOVA. $P<0.05$ was considered statistically significant.

\section{RESULTS}

\section{Precision}

The coefficients of variation (standard deviation as a percentage of the mean) for all assays are summarized in Table 2. All \% CVs 
Table 2. Intra- and inter-assay precision of the Aptima and cobas 6800 assays

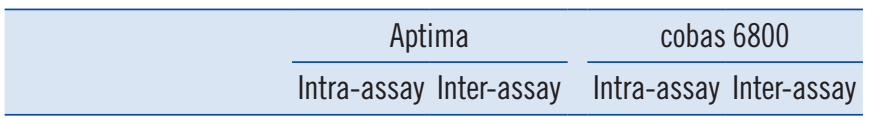

Low-concentration positive control

HIV-1

$\begin{array}{lllll}\text { Mean (log copies/mL) } & 2.91 & 2.91 & 2.38 & 2.35 \\ \text { SD (log copies/mL) } & 0.06 & 0.09 & 0.07 & 0.09 \\ \text { CV }(\%) & 1.90 & 3.10 & 2.80 & 3.90\end{array}$

HBV

$\begin{array}{lllll}\text { Mean }(\log I \mathrm{U} / \mathrm{mL}) & 2.77 & 2.77 & 1.75 & 1.64 \\ \text { SD }(\log I \mathrm{IU} / \mathrm{mL}) & 0.04 & 0.06 & 0.08 & 0.07 \\ \text { CV }(\%) & 1.35 & 2.21 & 4.71 & 4.32\end{array}$

HCV

$\begin{array}{lllll}\text { Mean }(\log \mathrm{IU} / \mathrm{mL}) & 2.32 & 2.32 & 2.93 & 2.96 \\ \mathrm{SD}(\log \mathrm{IU} / \mathrm{mL}) & 0.06 & 0.06 & 0.05 & 0.04 \\ \mathrm{CV}(\%) & 2.58 & 2.58 & 1.69 & 1.48\end{array}$

High-concentration positive control

HIV-1

\begin{tabular}{lllll} 
Mean (log copies/mL) & 5.20 & 5.20 & 5.51 & 5.51 \\
SD (log copies/mL) & 0.04 & 0.06 & 0.06 & 0.07 \\
CV (\%) & 0.80 & 1.10 & 1.18 & 1.29 \\
HBV & & & & \\
Mean (log IU/mL) & 4.50 & 4.50 & 5.34 & 5.27 \\
SD (log IU/mL) & 0.04 & 0.05 & 0.08 & 0.11 \\
CV (\%) & 0.87 & 1.11 & 1.43 & 2.11 \\
HCV & & & & \\
Mean (log IU/mL) & 5.41 & 5.41 & 7.01 & 7.03 \\
SD (log IU/mL) & 0.07 & 0.10 & 0.04 & 0.04 \\
CV (\%) & 1.33 & 1.76 & 0.61 & 0.52 \\
\hline
\end{tabular}

were within 5\%, indicating good assay precision, for all three viruses. For the Aptima assays, the inter-assay \% CV values were slightly higher than the corresponding intra-assay values.

For the cobas 6800 assays, the \% CV values for HIV-1 and HBV of the low-concentration positive control material were high when compared with those for HCV of low- and high-concentration positive control materials of the three viruses. For both lowand high-concentration positive control materials, the Aptima assays showed lower \% CV values in the HIV-1 and HBV assays than did cobas 6800, whereas the cobas $6800 \mathrm{HCV}$ assay yielded a lower \% CV than the Aptima assays.
Table 3. Agreement between the results obtained using the Aptima and cobas 6800 assays in terms of detection and quantitation of HIV-1, HBV, and HCV in clinical plasma samples

\begin{tabular}{llrrrr}
\hline & \multirow{2}{*}{ cobas 6800 } & \multicolumn{4}{c}{ Aptima } \\
\cline { 3 - 6 } & & TND & $<$ LLOQ & Quantitated & Total \\
\hline HIV-1 & TND & 89 & 11 & 0 & 100 \\
& $<$ LL0Q & 0 & 0 & 0 & 0 \\
& Quantitated & 2 & 3 & 196 & 201 \\
& Total & 91 & 14 & 196 & 301 \\
HBV & TND & 71 & 28 & 1 & 100 \\
& $<$ LLOQ & 0 & 0 & 0 & 0 \\
& Quantitated & 1 & 17 & 182 & 200 \\
& Total & 72 & 45 & 183 & 300 \\
HCV & TND & 98 & 1 & 2 & 101 \\
& $<$ LLOQ & 1 & 3 & 2 & 6 \\
& Quantitated & 0 & 6 & 126 & 132 \\
& Total & 99 & 10 & 130 & 239
\end{tabular}

Abbreviations: TND, target not detected; $\angle L L O Q$, below the lower limit of quantitation.

\section{Linearity}

The linearity of quantitation as assessed using the AccuSpan linearity panels was excellent for the Aptima assays $\left(R^{2}=0.995\right.$ 0.996) (Fig. 1).

\section{Comparison of the Aptima and cobas 6800 assays using clinical samples}

For HIV-1, the between-assay agreement was $94.68 \%$ (285/301) ( $\kappa=0.89 ; 95 \% \mathrm{Cl}, 0.83-0.94$ ) (Table 3). Two samples were detected as quantitated (with 119 and 193 copies/mL, respectively) by the cobas 6800 assay and as TND by the Aptima assay. Three samples were detected as quantitated $(\mathrm{VL}=100,110$, and 133 copies/mL, respectively) by the cobas 6800 assay, whereas they were detected as $<$ LLOQ by the Aptima assay. Eleven samples were detected as $\angle$ LLOQ by the Aptima assay and as TND by the cobas 6800 assay. For 196/301 (84.05\%) samples quantitated by both methods, the VL values ranged from 1.41 to 6.39 log copies $/ \mathrm{mL}$ and from 1.54 to $6.54 \log$ copies $/ \mathrm{mL}$ in the cobas 6800 and Aptima assays, respectively. The HIV-1 results of both assays were strongly correlated $\left(R^{2}=0.97\right.$; Fig. $\left.2 A\right)$. Bland-Altman analysis indicated a mean difference in the titer (Aptima vs. cobas 6800$)$ of -0.27 log copies $/ \mathrm{mL}(95 \% \mathrm{Cl},-0.84-0.29$; Fig. 2B).

There were 49 patients with VL values of 50-200 copies $/ \mathrm{mL}$ in at least one assay (Supplemental Data Table S1). The agreement rate for samples with low VL values (38\%) was lower than 
Park Y, et al.

Aptima vs. cobas 6800 for HIV-1, HBV, and HCV

(4)
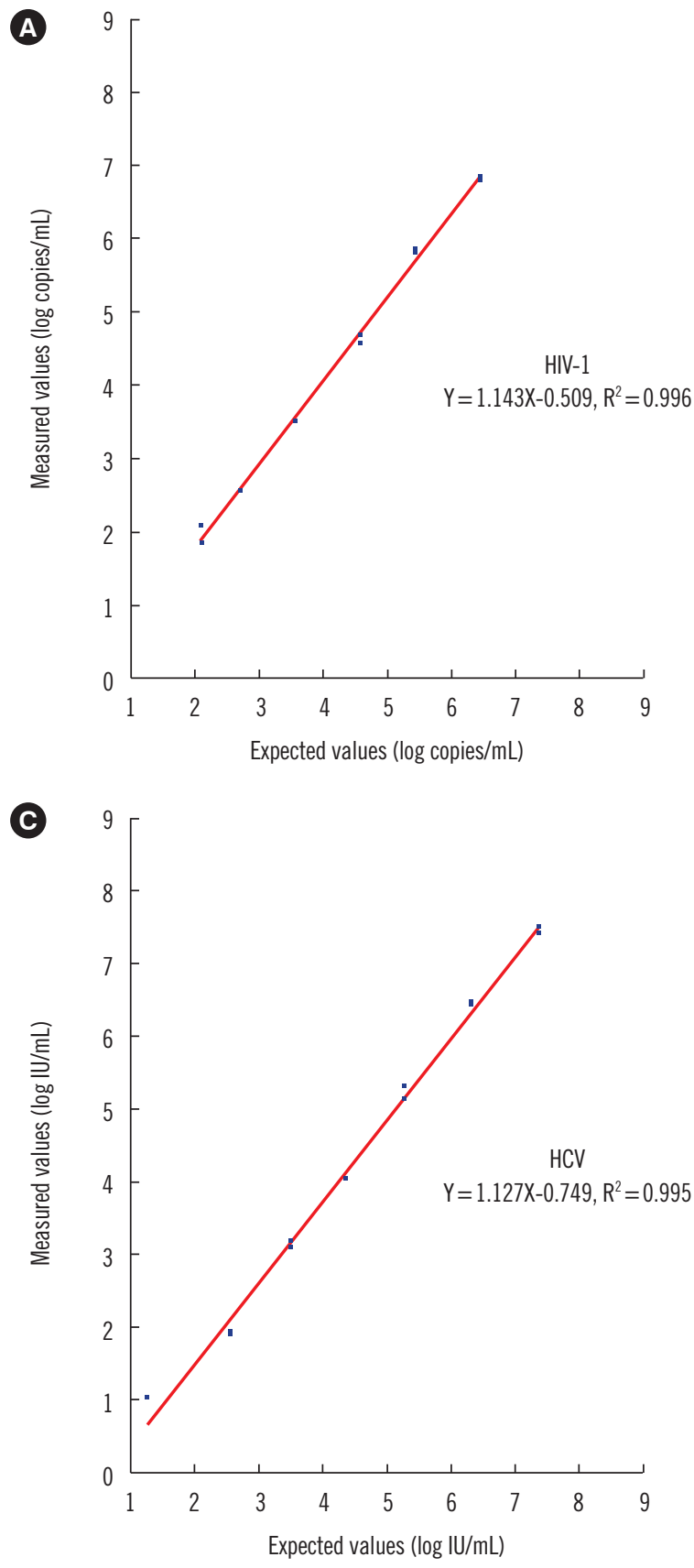

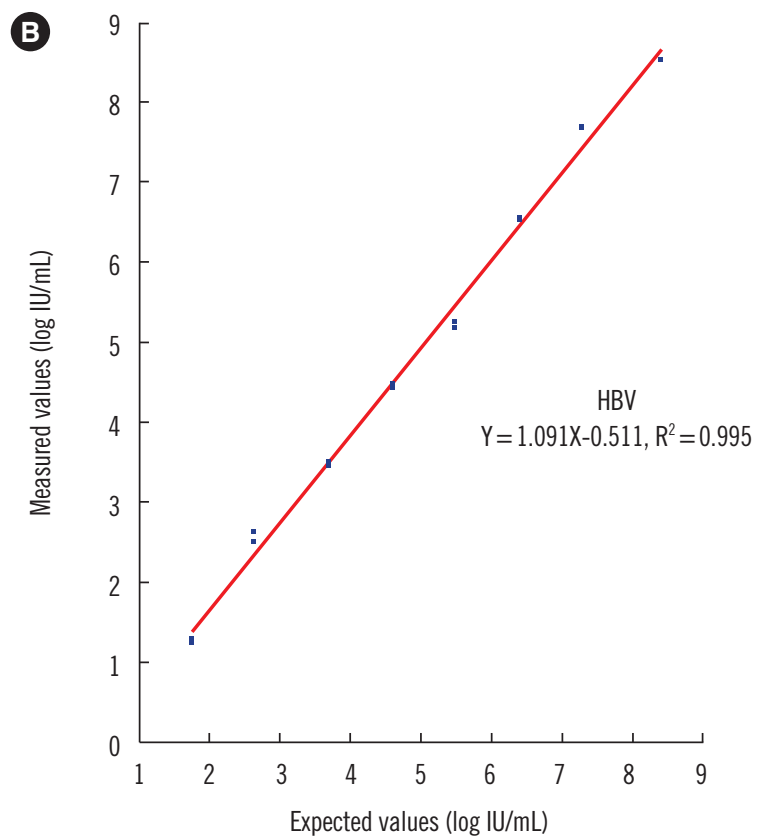

Fig. 1. Aptima results obtained with the AccuSpan Linearity Panels. The values are presented as log copies/mL for HIV-1 (A) and log IU/ $\mathrm{mL}$ for HBV (B) and HCV (C) (N=2 per target concentration level). that for the total samples (94.68\%). The detection rates of antiretroviral resistance mutations were similar among the VL groups, and no difference in VL values was observed between the detection ( $\mathrm{N}=19)$ and non-detection groups $(\mathrm{N}=13)$ (for cobas 6800 , the means \pm SDs for the detection and non-detection groups were $206.37 \pm 101.17$ and $241.62 \pm 151.90[P=0.44]$, respectively; the corresponding values in the Aptima assays were $116.79 \pm 169.58$ and $110.54 \pm 50.37[P=0.90]$, respectively). In the low $V L$ value range, we observed no association between treatment resistance mutations and increasing $\mathrm{VL}$ values; the presence of mutations associated with antiretroviral drug resistance may have been due to repeatedly high VL values (Supplemental Data Table S2).

The HBV agreement rate between the assays was $84.33 \%$ (253/300) ( $\kappa=0.69$; 95\% Cl, 0.63-0.76). One sample detected as quantitated by the cobas 6800 assay was detected as TND by the Aptima assay, and one sample detected as quantitated by the Aptima assay was detected as TND by the cobas 6800 

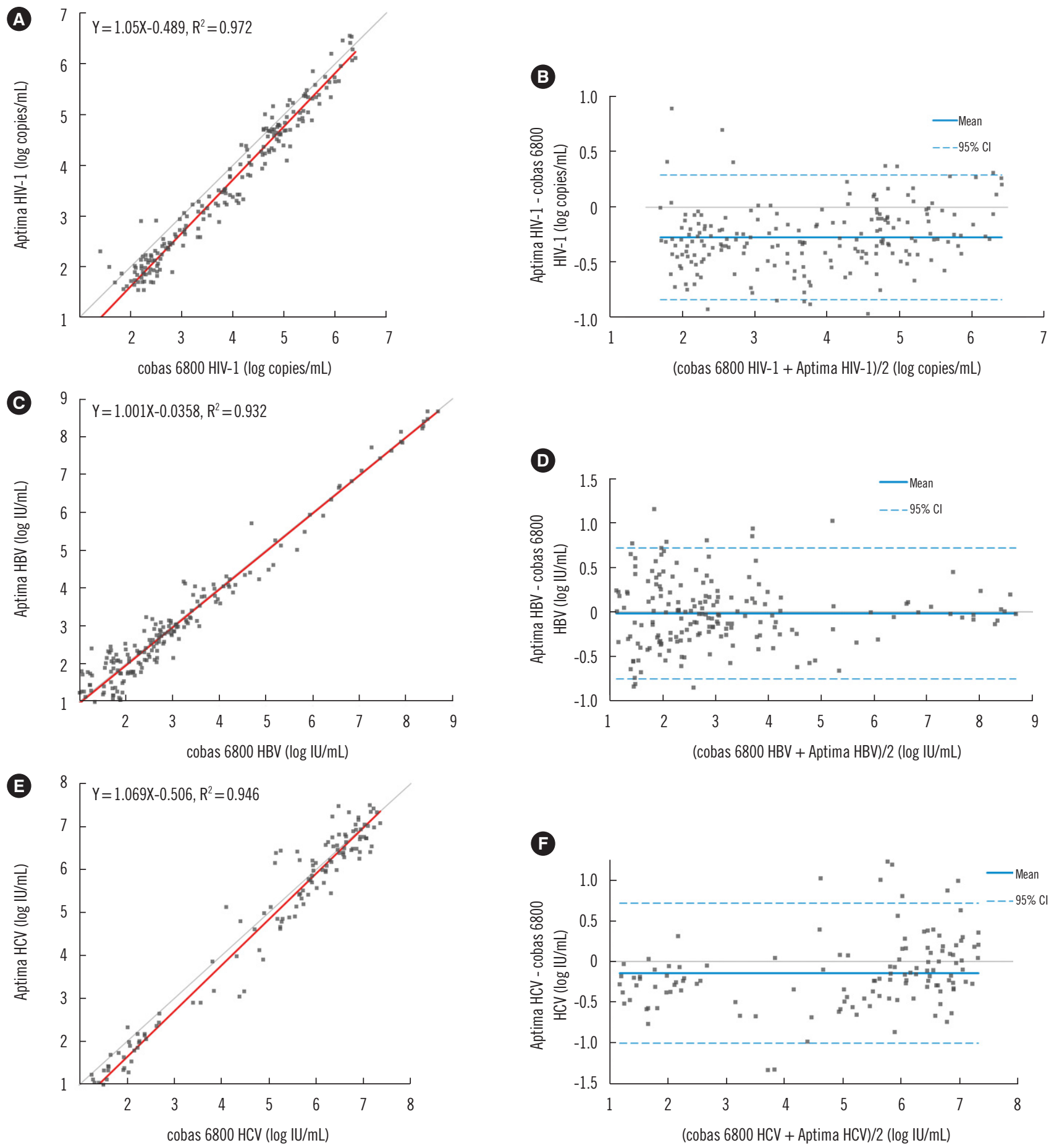

Fig. 2. Method comparison for HIV-1 ( $=196)$, HBV $(N=182)$, and HCV ( $=126)$ VL assessments. (A) Passing-Bablok regression results for 196 quantitated HIV-1 clinical samples that were assayed with the Aptima and cobas 6800 assays. (B) Bland-Altman plot of the difference between the Aptima and cobas 6800 HIV results vs. the mean. The mean bias was -0.27 log copies $/ \mathrm{mL}$, with $95 \% \mathrm{Cls}$ ranging from -0.84 to 0.29 log copies/mL. (C) Passing-Bablok regression for 182 quantitated HBV clinical samples that were assayed with both the Aptima and cobas 6800 assays. (D) Bland-Altman plot of the differences between the Aptima and cobas 6800 HBV results vs. the mean. The mean bias was -0.02 log copies $/ \mathrm{mL}$, with $95 \%$ Cls ranging from -0.75 to 0.71 log copies $/ \mathrm{mL}$. (E) Passing-Bablok regression results for 126 quantitated HCV clinical samples that were assayed with the Aptima and cobas 6800 assays. (F) Bland-Altman plot of the differences between the Aptima and cobas $6800 \mathrm{HCV}$ results vs. the mean. The mean bias was $-0.14 \mathrm{log}$ copies $/ \mathrm{mL}$, with $95 \% \mathrm{Cls}$ ranging from -1.00 to 0.72 log copies $/ \mathrm{mL}$.

Abbreviation: $\mathrm{Cl}$, confidence interval. 
Table 4. Analytical sensitivity of the Aptima assays assessed using clinical samples at different target concentrations

\begin{tabular}{lccc}
\hline & $\begin{array}{c}\text { Concentration } \\
\text { of target }\end{array}$ & $\begin{array}{c}\text { Detected } \\
\mathrm{N}(\%)\end{array}$ & $\begin{array}{c}\text { Quantitated } \\
\mathrm{N}(\%)\end{array}$ \\
\hline HIV-1 (copies/mL) & 20 & $20(100)$ & $11(55)$ \\
& 30 & $20(100)$ & $20(100)$ \\
HBV (IU/mL) & 40 & $20(100)$ & $20(100)$ \\
& 5 & $19(95)$ & $4(20)$ \\
& 7.5 & $20(100)$ & $3(15)$ \\
HCV (IU/mL) & 10 & $20(100)$ & $10(50)$ \\
& 15 & $20(100)$ & $14(70)$ \\
& 5 & $20(100)$ & $10(50)$ \\
& 7.5 & $20(100)$ & $13(65)$ \\
& 10 & $20(100)$ & $17(85)$ \\
& 15 & $20(100)$ & $19(95)$ \\
\hline
\end{tabular}

assay. Both samples had low VL values (17 and $26 \mathrm{IU} / \mathrm{mL}$ ). Seventeen samples quantitated by the cobas 6800 assay and detected as <LLOQ by the Aptima assay had a median VL value of $16.10 \mathrm{IU} / \mathrm{mL}$ (first quartile, $14.63 \mathrm{IU} / \mathrm{mL}$; third quartile, 29.37 $\mathrm{IU} / \mathrm{mL}$; minimum, $10.3 \mathrm{IU} / \mathrm{mL}$; maximum, $104.0 \mathrm{IU} / \mathrm{mL}$ ). Most $V L$ values were below the mean value $(28.62 \mathrm{IU} / \mathrm{mL})$, except for four results. Twenty-eight samples were detected as $<$ LLOQ by the Aptima assay and as TND by the cobas 6800 assay. For $182 / 300(60.67 \%)$ samples quantitated by both assays, the VL values ranged from 1.01 to $8.69 \mathrm{log} \mathrm{IU} / \mathrm{mL}$ and from 1.00 to $8.66 \mathrm{log} \mathrm{IU} / \mathrm{mL}$ in the cobas 6800 and Aptima assays, respectively. The HBV results of both assays were strongly correlated $\left(R^{2}=0.93\right.$; Fig. $\left.2 C\right)$. Bland-Altman analysis indicated a mean difference in titer (Aptima vs. cobas 6800) of -0.02 log copies/ $\mathrm{mL}(95 \% \mathrm{Cl},-0.75-0.71$; Fig. 2D).

The HCV agreement rate between the assays was $94.98 \%$ (227/239) ( $\kappa=0.90 ; 95 \% \mathrm{Cl}, 0.85-0.96$ ). Four samples quantitated with the Aptima assay yielded VL values near the LLOQ value $(19,16,16$, and $10 \mathrm{IU} / \mathrm{mL})$. The cobas 6800 assay yielded two samples as TND and two as $<$ LLOQ. Six samples were detected as quantitated by the cobas 6800 assay and as $<$ LLOQ by the Aptima assay. Their VL values were 15.1, 17.2, 18.4, 32.9, 42.1, and $42.8 \mathrm{IU} / \mathrm{mL}$. For $126 / 239$ (52.72\%) samples quantitated by both assays, the VL values ranged from 1.26 to $7.35 \mathrm{log} \mathrm{IU} / \mathrm{mL}$ and from 1.00 to $7.49 \mathrm{log} \mathrm{IU} / \mathrm{mL}$ in the cobas 6800 and Aptima assays, respectively. The HCV results of both assays were strongly correlated ( $R^{2}$ of 0.95 , Fig. $2 E$ ). Bland-Altman analysis indicated a mean difference in titer (Aptima vs. cobas 6800 ) of $-0.14 \log$ copies $/ \mathrm{mL}(95 \% \mathrm{Cl},-1.00-0.72$; Fig.
2F). Genotype information was available for 80/126 samples quantitated by both methods, with the following distribution: genotypes $1 \mathrm{a}, 1 \mathrm{~b}$, and 2 were detected in 1 (1.3\%), 35 (44.8\%), and $44(55.0 \%)$ samples, respectively. The mean difference between the Aptima and cobas 6800 assays in terms of HCV values was similar for genotypes $1 \mathrm{~b}$ and $2(0.107 \mathrm{log} \mathrm{IU} / \mathrm{mL}, 95 \% \mathrm{Cl},-0.057-$ 0.270 ; and $0.050 \log \mathrm{IU} / \mathrm{mL}, 95 \% \mathrm{Cl},-0.103-0.202$; respectively; $P=0.681$ ) (Supplemental Data Figure S1).

\section{Analytical sensitivity}

The overall detection rates of the Aptima assays for the three viruses were similar (Table 4). The VLs yielding a 100\% detection rate of HIV-1, HBV, and HCV were 20 copies $/ \mathrm{mL}, 7.5 \mathrm{IU} / \mathrm{mL}$, and $5 \mathrm{IU} / \mathrm{mL}$, respectively. Although the HIV-1 assay quantitated $100 \%$ of the replicates at $30 \mathrm{copies} / \mathrm{mL}$ (a value similar to the LLOQ reported by the manufacturer), the HBV and HCV assays quantitated $50 \%$ and $85 \%$ of the replicates at $10 \mathrm{IU} / \mathrm{mL}$, respectively.

\section{Cross-reactivity}

None of the viruses examined were detected by the Aptima assays. There was no evidence of cross-reactivity with other clinically important viruses.

\section{DISCUSSION}

The consistency of the assay results obtained from different laboratories and with different assays is paramount to the effective management of patients with chronic viral infections that require long-term treatment and monitoring [11-13]. The results produced by the cobas 6800 and Aptima assays for HIV-1 and HCV showed excellent agreement, and we found substantial concurrence between the assays for HBV, consistent with the results of previous studies [9, 11, 31, 32]. Overall, the results obtained with the cobas 6800 assays were slightly higher than those obtained with the Aptima assays, and the mean bias values for the three viruses were $<-0.3 \log$.

Similarly, the results produced by the cobas 6800 and Aptima assays showed excellent agreement for the detection/non-detection of HIV-1 RNA in clinical samples $(\kappa=0.89)$, and there was a good agreement between values obtained in samples quantitated by both assays (mean bias of $-0.27 \mathrm{log}$ ). Five HIV-1 samples were detected as quantitated by the cobas 6800 assay and as TND or $<$ LLOQ by the Aptima assay. Two of these five samples retained values below the lower LOD throughout the monitoring period. The samples detected as $<$ LLOQ by the Aptima 
assay had values lower than those detected by the cobas 6800 assay. A viral blip, which is an isolated detectable VL observed after virologic suppression that returns to virologic suppression or unconfirmed result upon repeated testing, may have occurred in these samples [11]. Blips are not associated with treatment failure and are generally considered an artifact caused by random assay variation rather than clinically significant elevations in viremia [11].

Current therapy guidelines for HIV-1 define virologic failure as a $V L$ repeatedly exceeding 200 copies/mL $[1,6,10]$. The present cases were not associated with treatment failure or a need to change treatment. Patients with VL values of 50-200 copies/ $\mathrm{mL}$ may continue with the same treatment with more frequent monitoring; there is no consensus on how to manage patients with $\mathrm{VL}$ values in this range $[1,6]$. The risk of emerging treatment resistance is likely low $[1,6,10]$. In such cases, confirming that VL values remain above the lower LOD and assessing treatment adherence and possible drug-drug or drug-food interactions are recommended.

The results produced by the cobas 6800 and Aptima assays showed good agreement for the detection/non-detection of HBV DNA in clinical samples $(\kappa=0.69)$ and the values obtained from samples quantitated by both assays (mean bias of $-0.02 \mathrm{log}$ ). Samples detected as <LLOQ by the Aptima HBV assay (45/300, $15 \%$ ) were either classified as TND or quantitated by the cobas 6800 assay, resulting in a decrease in the estimate of inter-assay agreement. Twenty-eight samples were detected by the Aptima assay alone, which might have been due to the amplification of HBV RNA [32]. The VL values of the other samples were near the LLOQ (median VL of $16.10 \mathrm{IU} / \mathrm{mL}$ ), and most were within the $\%$ CV of inter-assay precision.

Current guidelines for the monitoring of HCV-positive patients undergoing treatment recommend the use of a highly sensitive quantitative real-time HCV RNA assay with a LOD of $15 \mathrm{IU} / \mathrm{mL}$ and a LLOQ of $25 \mathrm{IU} / \mathrm{mL}$; both the cobas 6800 and Aptima assays comply with these requirements $[4,5,7]$. Both assays had an excellent agreement for the detection/non-detection of $\mathrm{HCV}$ RNA in clinical samples $(\kappa=0.90)$ and a good agreement of values in the samples quantitated by both assays (mean bias of -0.14 log). VL values of HCV genotype 3 have been associated with a high rate of under-reporting and high variance between assays $[9,14,26]$. The HCV genotypes detected were $1 a(N=1)$, $1 b(N=35)$, and $2(N=44)$; there was no difference or significant variation in VL values between patients with genotypes $1 \mathrm{~b}$ and 2 (Supplemental Data Figure S1).

In the quantitative comparison of the cobas 6800 and Aptima assays, several samples detected as $\angle \mathrm{LLOQ}$ or TND by one assay were quantitated by the other, with respect to HIV-1, HBV, and HCV VL values. In samples with VL values near the LLOQ, even those within the \% CV of the allowable inter-assay precision, inconsistent results may be observed. As the two assays use different principles (TMA and real-time PCR), the probability of inconsistent results is higher than that observed for assays using the same principle. In addition, as the target regions of HIV-1 and HBV were different, more discrepancies were observed for these viruses than for HCV. Different LODs and linearity ranges may have contributed to the discrepancy in the results.

In the analytical sensitivity assessment, the lowest concentrations required for a $100 \%$ detection rate were 20 copies $/ \mathrm{mL}, 7.5$ $\mathrm{IU} / \mathrm{mL}$, and $5.0 \mathrm{IU} / \mathrm{mL}$ for HIV-1, HBV, and HCV, respectively, which are higher than the LODs reported by the manufacturer of the Aptima assays. Accurate assessment of the LOD requires precise dilutions close to the LOD concentration. For HBV and $\mathrm{HCV}$, the quantitation rates were $50 \%$ and $85 \%$, respectively, at the LLOQ level. The samples used for analytical sensitivity assessment were selected based on the cobas 6800 results, and upon comparison, the VL values of the Aptima assays were lower than those of cobas 6800, which we suspect to have influenced the low quantitation rate.

This study had some limitations. As the cobas 6800 system is routinely used in our laboratory, we collected the samples based on the assay results produced by this system, which may have affected the comparisons. The linearity and cross-reactivity were evaluated using the Aptima assays. HCV genotypes 3, 4, 5, and 6 were not detected. Future studies should compare the cobas 6800 and Aptima assays using samples assayed in parallel and assess assay sensitivity in HCV detection using samples with a wider range of genotypes than those detected in this study.

In summary, both the cobas 6800 and Aptima assays show good analytical performance for the determination of HIV-1, HBV, and HCV VL values. The results of this study, including the estimates of the correlation and agreement between the assay results obtained with these assays, suggest that the two assays can be used interchangeably for the long-term monitoring of chronic infections. Clinical laboratories should base their choice of assay system on factors such as the assay principle and type and the timeline for obtaining results.

\section{ACKNOWLEDGEMENTS}

This study was supported by Anse (Seongnam-si, Gyeonggi-do, Korea), Korean representative of Hologic, San Diego, CA, USA. 


\section{AUTHOR CONTRIBUTIONS}

Park Y: Formal analysis, Writing - Original draft, Reviewing and Editing; Kim S: Conceptualization, Methodology, Formal analysis, Writing - Reviewing and Editing, Supervision, Project administration; Roh J: Data collection, Resource acquisition. All authors take responsibility for the intellectual content of this manuscript and have approved its submission.

\section{CONFLICTS OF INTEREST}

No potential conflicts of interest relevant to this study are reported.

\section{RESEARCH FUNDING}

None.

\section{ORCID}

Younhee Park https://orcid.org/0000-0001-8458-1495

Juhye Roh

Sinyoung Kim https://orcid.org/0000-0003-0078-1145 https://orcid.org/0000-0002-2609-8945

\section{REFERENCES}

1. World Health Organization. Consolidated guidelines on the use of antiretroviral drugs for treating and preventing HIV infection: recommendations for a public health approach, 2nd ed [Internet]. Geneva: World Health Organization; 2016 [cited 2021 Dec 12]. Available from: https:// www.who.int/publications/i/item/9789241549684

2. AASLD/IDSA HCV Guidance Panel. Hepatitis C guidance: AASLD-IDSA recommendations for testing, managing, and treating adults infected with hepatitis C virus. Hepatology 2015;62:932-54.

3. European Association for the Study of the Liver. Electronic address: easloffice@easloffice.eu, European Association for the Study of the Liver. EASL 2017 Clinical Practice Guidelines on the management of hepatitis B virus infection. J Hepatol 2017;67:370-98.

4. European Association for the Study of the Liver. Electronic address: easloffice@easloffice.eu; European Association for the Study of the Liver. EASL Recommendations on Treatment of Hepatitis C 2018. J Hepatol 2018;69:461-511.

5. European Association for the Study of the Liver. Electronic address: easloffice@easloffice.eu; Clinical Practice Guidelines Panel: Chair:, EASL Governing Board representative:, Panel members. EASL recommendations on treatment of hepatitis C: final update of the series is. J Hepatol 2020; 73:1170-218.

6. Ryom L, Boesecke C, Bracchi M, Ambrosioni J, Pozniak A, Arribas J, et al. Highlights of the 2017 European AIDS Clinical Society (EACS) Guidelines for the treatment of adult HIV-positive persons version 9.0. HIV Med 2018;19:309-15

7. Terrault NA, Bzowej NH, Chang KM, Hwang JP, Jonas MM, Murad MH. AASLD guidelines for treatment of chronic hepatitis B. Hepatology 2016;
63:261-83.

8. Terrault NA, Lok ASF, McMahon BJ, Chang KM, Hwang JP, Jonas MM, et al. Update on prevention, diagnosis, and treatment of chronic hepatitis B: AASLD 2018 hepatitis B guidance. Hepatology 2018;67:1560-99.

9. Aretzweiler G, Leuchter S, García-Álvarez M, Simon C, Marins E, Paxinos $E$, et al. Analytical performance of four molecular platforms used for HIV-1, HBV and HCV viral load determinations. Expert Rev Mol Diagn 2019;19:941-9.

10. Günthard HF, Aberg JA, Eron JJ, Hoy JF, Telenti A, Benson CA, et al. Antiretroviral treatment of adult HIV infection: 2014 recommendations of the International Antiviral Society-USA Panel. Jama 2014;312:41025.

11. Wiesmann F, Ehret R, Naeth G, Däumer M, Fuhrmann J, Kaiser R, et al. Multicenter evaluation of two next-generation HIV-1 quantitation assays, Aptima Quant Dx and cobas 6800, in comparison to the RealTime HIV-1 reference assay. J Clin Microbiol 2018;56.

12. Wiesmann F, Naeth G, Sarrazin C, Berger A, Kaiser R, Ehret R, et al. Variation analysis of six HCV viral load assays using low viremic HCV samples in the range of the clinical decision points for HCV protease inhibitors. Med Microbiol Immunol 2015;204:515-25.

13. Hayden RT, Sun Y, Tang L, Procop GW, Hillyard DR, Pinsky BA, et al. Progress in quantitative viral load testing: variability and impact of the WHO quantitative international standards. J Clin Microbiol 2017;55:42330.

14. Schalasta G, Speicher A, Börner A, Enders M. Performance of the new Aptima HCV Quant Dx assay in comparison to the Cobas TaqMan HCV2 test for use with the high pure system in detection and quantification of hepatitis C virus RNA in plasma or serum. J Clin Microbiol 2016;54:1101-7.

15. Manak MM, Hack HR, Nair SV, Worlock A, Malia JA, Peel SA, et al. Evaluation of Hologic Aptima HIV-1 Quant Dx assay on the Panther system on HIV subtypes. J Clin Microbiol 2016;54:2575-81.

16. Nair SV, Kim HC, Fortunko J, Foote T, Peling T, Tran C, et al. Aptima HIV1 Quant Dx-A fully automated assay for both diagnosis and quantification of HIV-1. J Clin Virol 2016;77:46-54.

17. Sauné K, Raymond S, Boineau J, Pasquier C, Izopet J. Detection and quantification of HIV-1 RNA with a fully automated transcription-mediated-amplification assay. J Clin Virol 2016;84:70-3.

18. Schalasta G, Börner A, Speicher A, Enders M. Comparative evaluation of the Aptima HIV-1 Quant Dx assay and COBAS TaqMan HIV-1 v2.0 assay using the Roche High Pure System for the quantification of HIV-1 RNA in plasma. Clin Chem Lab Med 2016;54:493-9.

19. Tabrizi SN, Costa AM, Su J, Lowe P, Bradshaw CS, Fairley CK, et al. Evaluation of the Hologic Panther transcription-mediated amplification assay for detection of Mycoplasma genitalium. J Clin Microbiol 2016;54: 2201-3.

20. Chevaliez S, Dauvillier C, Dubernet F, Poveda JD, Laperche S, Hézode C, et al. The new Aptima HBV Quant Real-Time TMA assay accurately quantifies hepatitis B virus DNA from genotypes A to F. J Clin Microbiol 2017; 55:1211-9

21. Chevaliez S, Dubernet F, Dauvillier C, Hézode C, Pawlotsky JM. The new Aptima HCV Quant Dx Real-time TMA assay accurately quantifies hepatitis C virus genotype 1-6 RNA. J Clin Virol 2017;91:5-11.

22. Schønning K, Johansen K, Landt B, Benfield T, Westh H. Comparison of the Hologic Aptima HIV-1 Quant Dx assay to the Roche COBAS Ampliprep/COBAS TaqMan HIV-1 test v2.0 for the quantification of HIV-1 RNA in plasma samples. J Clin Virol 2017;92:14-9.

23. Schønning K, Pedersen MS, Johansen K, Landt B, Nielsen LG, Weis N, et al. Analytical and clinical performance of the Hologic Aptima HCV Quant Dx assay for the quantification of HCV RNA in plasma samples. J Virol Methods 2017;248:159-65. 
24. Worlock A, Blair D, Hunsicker M, Le-Nguyen T, Motta C, Nguyen C, et al. Analytical characteristics and comparative evaluation of Aptima HCV Quant Dx assay with the Abbott RealTime HCV assay and Roche COBAS AmpliPrep/COBAS TaqMan HCV quantitative test v2.0. Virol J 2017; 14:66.

25. Longo S, Bon I, Musumeci G, Bertoldi A, D’Urbano V, Calza L, et al. Comparison of the Aptima HIV-1 Quant Dx assay with the COBAS AmpliPrep/COBAS TaqMan HIV-1 v2.0 test for HIV-1 viral load quantification in plasma samples from HIV-1-infected patients. Health Sci Rep 2018;1: e31.

26. May S, Adamska E, Tang JW. Evaluating the aptima HIV-1 quant Dx, HCV quant Dx and HBV quant assays against the Abbott HIV-1, HCV and HBV RealTime assays. J Clin Virol 2018;106:7-10.

27. Schalasta G, Börner A, Speicher A, Enders M. Evaluation of the Aptima HBV Quant assay vs. the COBAS TaqMan HBV test using the high pure system for the quantitation of HBV DNA in plasma and serum samples. Clin Chem Lab Med 2018;56:634-41.

28. Schønning K, Johansen K, Nielsen LG, Weis N, Westh H. Analytical performance of the Hologic Aptima HBV Quant assay and the COBAS Ampliprep/COBAS TaqMan HBV test v2.0 for the quantification of HBV DNA in plasma samples. J Clin Virol 2018;104:83-8.
29. Mwau M, Mbugua J, Danda J, Handa A, Worlock A, Kim HC, et al. Performance evaluation of the Aptima HIV-1 Quant Dx assay for detection of HIV in infants in Kenya. J Clin Virol 2020;125:104289.

30. Rossetti R, Smith T, Luo W, Taussig J, Valentine-Graves M, Sullivan P, et al. Performance evaluation of the Aptima HIV-1 RNA Quant assay on the Panther system using the standard and dilution protocols. J Clin Virol 2020;129:104479.

31. Aretzweiler G, Leuchter S, Simon CO, Marins E, Frontzek A. Generating timely molecular diagnostic test results: workflow comparison of the cobas ${ }^{\circledR} 6800 / 8800$ to Panther. Expert Rev Mol Diagn 2019;19:951-7.

32. Maasoumy Bz, Geretti AM, Frontzek A, Austin H, Aretzweiler G, GarciaÁlvarez M, et al. HBV-RNA co-amplification may influence HBV DNA viral load determination. Hepatol Commun 2020;4:983-97.

33. CLSI. Evaluation of precision of quantitative measurement procedure; approved guideline. 3rd ed. EP05A3. Wayne, PA: Clinical Laboratory Standards Institute, 2014.

34. CLSI. Evaluation of the linearity of quantitative measurement procedures: a statistical approach; approved guideline. EP06-A. Wayne, PA: Clinical and Laboratory Standards Institute, 2003.

35. Stanford University HIV Drug Resistance Database. HIVdb version 9.0. https://hivdb.stanford.edu/hivdb/ (Updated on Feb 2021). 
Park Y, et al.

Aptima vs. cobas 6800 for HIV-1, HBV, and HCV

Supplemental Data Table S1. Agreement between the Aptima and cobas 6800 assays for low viremia levels of HIV-1

\begin{tabular}{lcccc}
\hline \multirow{2}{*}{ cobas 6800 } & \multicolumn{4}{c}{ Aptima } \\
\cline { 2 - 5 } & LLOQ $<50$ & $50-200$ & $>200$ & Total \\
\hline LLOQ<50 & 0 & 1 & 1 & 2 \\
$50-200$ & 5 & 19 & 1 & 25 \\
$>200$ & 0 & 23 & 0 & 23 \\
Total & 5 & 43 & 2 & 50 \\
\hline
\end{tabular}

Abbreviation: LLOQ, lower limit of quantitation. 
Supplemental Data Table S2. Agreement between the Aptima and cobas 6800 assays for antiretroviral drug resistance mutation results of HIV-1

\begin{tabular}{lccc}
\hline \multicolumn{2}{c}{ Detected number/Not detected number of drug resistance mutation $(\mathrm{N}=32)$} \\
\cline { 2 - 4 } cobas 6800 & \multicolumn{3}{c}{ Aptima } \\
\hline $\mathrm{LLOQ}<50$ & $50-200$ & $>200$ \\
\hline LLOQ $<50$ & $0 / 0$ & $0 / 0$ & $0 / 0$ \\
$>200$ & $2 / 1$ & $7 / 5$ & $1 / 0$ \\
\hline
\end{tabular}

Abbreviation: LLOQ, lower limit of quantitation. 
Park Y, et al.

Aptima vs. cobas 6800 for HIV-1, HBV, and HCV
ANNALS OF

LABORATORY

MEDICINE

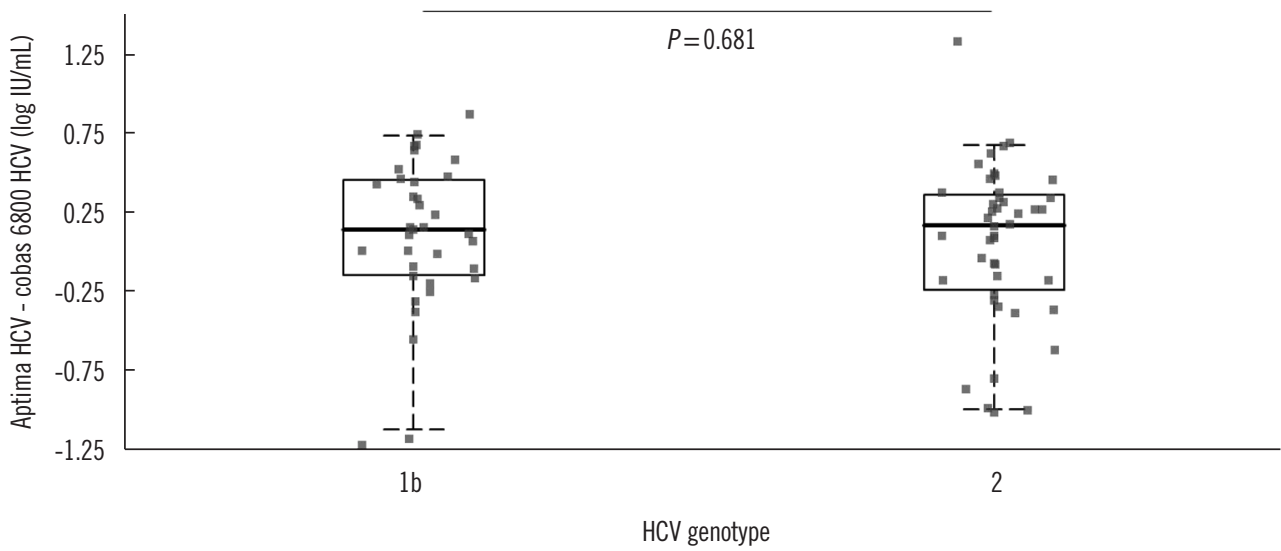

Supplemental Data Figure S1. HCV genotype (genotype 1b, $\mathrm{N}=35$; genotype 2, $\mathrm{N}=44$ ) and difference in HCV results between the Aptima and cobas 6800 assays.

Abbreviation: $\mathrm{HCV}$, hepatitis $\mathrm{C}$ virus. 